\title{
Dynamic behaviors of a nonautonomous modified Leslie-Gower predator-prey model with Holling-type III schemes and a prey refuge
}

\author{
Fengde Chen ${ }^{\mathrm{a}}$, Qiaoxia Lin ${ }^{\mathrm{a}}$, Xiangdong Xie ${ }^{\mathrm{b}, *}$, Yalong Xue ${ }^{\mathrm{b}}$ \\ ${ }^{a}$ College of Mathematics and Computer Science, Fuzhou University, Fuzhou, Fujian, 350002, P. R. China. \\ ${ }^{b}$ Department of Mathematics, Ningde Normal University, Ningde, Fujian, 352300, P. R. China.
}

\begin{abstract}
A nonautonomous modified Leslie-Gower predator-prey model with Holling-type III schemes and a prey refuge is proposed and studied in this paper. Sufficient conditions which guarantee the permanence and global stability of the system are obtained, respectively. Our results indicate that the prey refuge has no influence on the persistent property of the system, while it has positive effect on the stability property of the system. Numeric simulations show the feasibility of the main results. (C) 2017 All rights reserved.
\end{abstract}

Keywords: Predator, prey, permanence, global stability. 2010 MSC: 34C25, 34D23, 92D25, 34D20, 34D40.

\section{Introduction}

Throughout this paper, for a bounded continuous function $g$ defined on $R$, let $g^{L}$ and $g^{M}$ be defined as

$$
g^{\mathrm{L}}=\inf _{\mathrm{t} \in[0,+\infty)} g(\mathrm{t}), \quad g^{\mathrm{M}}=\sup _{\mathrm{t} \in[0,+\infty)} \mathrm{g}(\mathrm{t}) .
$$

Traditional Leslie-Gower predator-prey model, which was proposed by Leslie $[17,18]$ takes the form:

$$
\frac{d H}{d t}=\left(r_{1}-a_{1} P-b_{1} H\right) H, \quad \frac{d P}{d t}=\left(r_{2}-a_{2} \frac{P}{H}\right) P,
$$

where $\mathrm{H}$ and $\mathrm{P}$ are the density of prey species and the predator species at time $t$, respectively. In this model, the "carrying capacity" of the predator's environment is proportional to the number of prey. Though at first sight the model seems very simple, its stability property was not investigated until 2001. By constructing a suitable Lyapunov function, Korobeinikov [15] showed the unique positive equilibrium of the system (1.1) is globally stable.

\footnotetext{
${ }^{*}$ Corresponding author

Email addresses: fdchen@263.net (Fengde Chen), 2285096851@qq.com (Qiaoxia Lin), latexfzu@126.com (Xiangdong Xie), 642286433@qq.com (Yalong Xue)

doi:10.22436/jmcs.017.02.08
} 
Aziz-Alaoui and Daher Okiye [2] argued that a suitable predator prey model should incorporate some kind of functional response, while the predator species could have other food resource. Basing on those assumption, they proposed a predator-prey model with modified Leslie-Gower and Holling-type II schemes as follow:

$$
\begin{aligned}
& \dot{x}(t)=x\left(r_{1}-b_{1} x-\frac{a_{1} y}{x+k_{1}}\right), \\
& \dot{y}(t)=y\left(r_{2}-\frac{a_{2} y}{x+k_{2}}\right) .
\end{aligned}
$$

Such topic as the boundedness and global stability of the system were investigated in [2].

Yang and Li [31] argued that Holling-type III response exists universally in population dynamics, and they proposed a system incorporating a modified version of the Leslie-Gower functional response as well as that of the Holling-type III functional response:

$$
\begin{aligned}
& \dot{x}(t)=x\left(a_{1}-b x-\frac{c_{1} x y}{x^{2}+k_{1}}\right), \\
& \dot{y}(t)=y\left(a_{2}-\frac{c_{2} y}{x+k_{2}}\right) .
\end{aligned}
$$

They investigated the local stability property of the above system. For more works on Leslie-Gower predator-prey model, one could refer to $[1,4,7,8,19,21,26,29,30,34-37]$ and the references cited therein.

On the other hand, more and more scholars paid attention to the dynamic behaviors of the predatorprey system incorporating a prey refuge. Kar and Misra [14] proposed the following prey-predator model with Holling type II response function incorporation a prey refuge:

$$
\begin{aligned}
& \frac{d x}{d t}=\alpha x\left(1-\frac{x}{k}\right)-\frac{\beta(1-m) x y}{1+a(1-m) x}, \\
& \frac{d y}{d t}=-\gamma y+\frac{c \beta(1-m) x y}{1+a(1-m) x} .
\end{aligned}
$$

Some interesting results about the existence of unique global asymptotical stable limit cycle were obtained.

Huang et al. [12] studied the influence of prey refuge on the prey-predator model with Holling type III functional response:

$$
\begin{aligned}
& \frac{d x}{d t}=a x-b x^{2}-\frac{\alpha(1-m)^{2} x^{2} y}{\beta^{2}+(1-m)^{2} x^{2}}, \\
& \frac{d y}{d t}=-c y+\frac{k \alpha(1-m)^{2} x^{2} y}{\beta^{2}+(1-m) x^{2}} .
\end{aligned}
$$

They investigated the existence of unique globally asymptotically stable limit cycle and the globally attractive positive equilibrium.

It is natural to investigate the influence of prey refuge on the Leslie-Gower type predator-prey model. Already, Chen et al. [7] extended the model (1.1) by incorporating a refuge protecting $\mathrm{mH}$ of the prey, where $m \in[0,1)$ is constant and proposed the following system:

$$
\frac{d H}{d t}=\left(r_{1}-b_{1} H\right) H-a_{1}(1-m) H P, \quad \frac{d P}{d t}=\left(r_{2}-a_{2} \frac{P}{(1-m) H}\right) P,
$$

where $m \in[0,1)$ and $r_{i}, a_{i}, i=1,2, b_{1}$ are all positive constants. They showed that the unique positive equilibrium of the system is globally stable, consequently, prey refuge has no influence on the persistent property of the system.

Corresponding to system (1.2) recently, Yue [36] proposed and studied the following modified LeslieGower predator-prey model with Holling-type II schemes and a prey refuge

$$
\dot{x}(t)=x\left(r_{1}-b_{1} x-\frac{a_{1}(1-m) y}{(1-m) x+k_{1}}\right),
$$




$$
\dot{y}(t)=y\left(r_{2}-\frac{a_{2} y}{(1-m) x+k_{2}}\right),
$$

where $x(t)$ and $y(t)$ denote the densities of the predator and prey species at time $t$, respectively and all the coefficients are all positive constants, $0 \leqslant \mathrm{~m}<1$. The author obtained a set of sufficient conditions which ensure the global attractivity of a positive equilibrium.

Now stimulate by the works of [7] and [36], it is natural to incorporate the prey refuge to the system (1.3) and investigate its dynamic behaviors, i.e., the following predator-prey system incorporating a modified version of the Leslie-Gower functional response as well as that of the Holling-type III functional response and a prey refuge:

$$
\begin{aligned}
& \dot{x}(t)=x\left(a_{1}-b x\right)-\frac{c_{1}(1-m)^{2} x^{2} y}{(1-m)^{2} x^{2}+k_{1}}, \\
& \dot{y}(t)=y\left(a_{2}-\frac{c_{2} y}{(1-m) x+k_{2}}\right) .
\end{aligned}
$$

Considering the biological and environmental periodicity change (i.e., seasonal effects of weather, food supplies, mating habit etc.), it is reasonable to study the system with time varying coefficients. Recently, many scholars (see $[5,6,10,11,13,16,20,22-25,27,28,32,33,38]$ ) studied the dynamics behaviors of the non-autonomous predator-prey system, and their success motivated us to study the non-autonomous case of the system (1.4), i.e.,

$$
\begin{aligned}
& \dot{x}(t)=x\left(a_{1}(t)-b(t) x\right)-\frac{c_{1}(t)(1-m(t))^{2} x^{2} y}{(1-m(t))^{2} x^{2}+k_{1}(t)}, \\
& \dot{y}(t)=y\left(a_{2}(t)-\frac{c_{2}(t) y}{(1-m(t)) x+k_{2}(t)}\right),
\end{aligned}
$$

where $x(t)$ and $y(t)$ denote the densities of the predator and prey species at time $t$, respectively, in system (1.5), we incorporating a refuge protecting $m(t) x$ of the prey, where $m(t) \in[0,1)$, this leaves $(1-m(t)) x$ of the prey available to the predator. All the other parameters have the same meaning as that of the system (1.3).

Throughout this paper, we assume that

$\left(H_{1}\right) k_{i}(t), c_{i}(t), a_{i}(t), i=1,2, m(t), b(t)$ are continuous and strictly positive functions, which satisfy

$$
\begin{gathered}
\min \left\{c_{i}^{L}, k_{i}^{L}, a_{i}^{L}, m^{L}, b^{L}\right\}>0, \\
\max \left\{c_{i}^{M}, k_{i}^{M}, a_{i}^{M}, m^{M}, b^{M}\right\}<+\infty .
\end{gathered}
$$

We consider (1.5) together with the following initial conditions

$$
x(0)>0, \quad y(0)>0 .
$$

It is not difficult to see that solutions of (1.5)-(1.6) are well-defined for all $t \geqslant 0$ and satisfy

$$
x(t)>0, \quad y(t)>0, \quad \forall t \geqslant 0 .
$$

Definition 1.1. If there exist positive constants $m_{1}, M_{1}, m_{2}$ and $M_{2}$, which are independent of the solutions of system (1.5) such that each solution $(x(t), y(t))^{T}$ of system (1.5) satisfies

$$
\begin{aligned}
& 0<m_{1} \leqslant \liminf _{t \rightarrow+\infty} x(t) \leqslant \limsup _{t \rightarrow+\infty} x(t) \leqslant M_{1}, \\
& 0<m_{2} \leqslant \liminf _{t \rightarrow+\infty} y(t) \leqslant \limsup _{t \rightarrow+\infty} y(t) \leqslant M_{2},
\end{aligned}
$$

then system (1.5) is permanent.

Definition 1.2. Let $(x(t), y(t))^{\top}$ and $\left(x_{1}(t), y_{1}(t)\right)^{\top}$ be any two positive solutions of system (1.5)-(1.6), if

$$
\lim _{t \rightarrow+\infty}\left(\left|x(t)-x_{1}(t)\right|+\left|y(t)-y_{1}(t)\right|\right)=0,
$$

then system (1.5) is globally attractive. 
As far as system (1.5) is concerned, the most important topic is to investigate the persistent and stability property of the system and to find out the influence of prey refuge on the dynamic behaviors of the system.

The paper is arranged as follows: In Section 2 we obtain sufficient conditions which guarantee the permanence of the system (1.5). In Section 3 we obtain sufficient conditions which ensure the global attractivity of the system (1.5). In Section 4 an example together with its numeric simulations to illustrate the feasibility of the main results. We end this paper by a briefly discussion as Section 5 . For more works on predator-prey system incorporating a prey refuge, one can refer to [23-29] and the references cited therein.

\section{Permanence}

Lemma 2.1 ([9]). If $\mathrm{a}>0, \mathrm{~b}>0$ and $\dot{x} \geqslant x(\mathrm{~b}-\mathrm{ax})$ when $\mathrm{t} \geqslant 0$ and $x(0)>0$, we have

$$
\liminf _{t \rightarrow+\infty} x(t) \geqslant \frac{b}{a} \text {. }
$$

If $\mathrm{a}>0, \mathrm{~b}>0$ and $\dot{\mathrm{x}} \leqslant x(\mathrm{~b}-\mathrm{ax})$ when $\mathrm{t} \geqslant 0$ and $x(0)>0$, we have

$$
\limsup _{t \rightarrow+\infty} x(t) \leqslant \frac{b}{a}
$$

Lemma 2.2. The domain $\left.R_{+}^{2}=\{x, y) \mid x>0, y>0\right\}$ is invariant with respect to (1.5).

Proof. Since

$$
x(t)=x(0) \exp \left\{\int_{0}^{t} \Delta_{1}(s) d s\right\}>0, \quad y(t)=y(0) \exp \left\{\int_{0}^{t} \Delta_{2}(s) d s\right\}>0,
$$

where

$$
\begin{aligned}
& \Delta_{1}(s) \stackrel{\text { def }}{=} a_{1}(s)-b(s) x(s)-\frac{c_{1}(s)(1-m(s))^{2} \chi(s) y(s)}{(1-m(s))^{2}(x(s))^{2}+k_{1}(s)}, \\
& \Delta_{2}(s) \stackrel{\text { def }}{=} a_{2}(s)-\frac{c_{2}(s) y(s)}{(1-m(s)) x+k_{2}(s)} .
\end{aligned}
$$

The assertion of the lemma follows immediately for all $t \in[0,+\infty)$.

Lemma 2.3. Let $(x(t), y(t))^{\top}$ be any solution of system (1.5)-(1.6), then

$$
\begin{aligned}
& \limsup _{t \rightarrow+\infty} x(t) \leqslant \frac{a_{1}^{M}}{b^{L}} \stackrel{\text { def }}{=} M_{1}, \\
& \limsup _{t \rightarrow+\infty} y(t) \leqslant \frac{a_{2}^{M}\left(\left(1-m^{L}\right) M_{1}+k_{2}^{M}\right)}{c_{2}^{L}} \stackrel{\text { def }}{=} M_{2} .
\end{aligned}
$$

Proof. Let $(x(t), y(t))^{\top}$ be any solution of system (1.5)-(1.6). From the first equation of system (1.5), it follows that

$$
\begin{aligned}
\dot{x}(t) & =x\left(a_{1}(t)-b(t) x\right)-\frac{c_{1}(t)(1-m(t))^{2} x^{2} y}{(1-m(t))^{2} x^{2}+k_{1}(t)} \\
& \leqslant x\left(a_{1}^{M}-b^{L} x\right) .
\end{aligned}
$$

Applying Lemma 2.1 to (2.1), it immediately follows that

$$
\limsup _{t \rightarrow+\infty} x(t) \leqslant \frac{a_{1}^{M}}{b^{L}} \stackrel{\text { def }}{=} M_{1} .
$$


For any positive constant $\varepsilon>0$ small enough, it follows from (2.2) that there exists a $T_{1}>0$ such that

$$
x(t)<M_{1}+\varepsilon, \quad \forall t \geqslant T_{1} .
$$

For $t>T_{1}$, (2.3) together with the second equation of system (1.5) leads to

$$
\begin{aligned}
\dot{y}(t) & =y\left(a_{2}(t)-\frac{c_{2}(t) y}{(1-m(t)) x+k_{2}(t)}\right) \\
& \leqslant y\left(a_{2}^{M}-\frac{c_{2}^{L} y}{\left(1-m^{L}\right)\left(M_{1}+\varepsilon\right)+k_{2}^{M}}\right) .
\end{aligned}
$$

Applying Lemma 2.1 to (2.4), it immediately follows that

$$
\limsup _{t \rightarrow+\infty} y(t) \leqslant \frac{a_{2}^{M}\left(\left(1-m^{L}\right)\left(M_{1}+\varepsilon\right)+k_{2}^{M}\right)}{c_{2}^{L}} .
$$

Setting $\varepsilon \rightarrow 0$, then

$$
\limsup _{t \rightarrow+\infty} y(t) \leqslant \frac{a_{2}^{M}\left(\left(1-m^{L}\right) M_{1}+k_{2}^{M}\right)}{c_{2}^{L}} \stackrel{\text { def }}{=} M_{2} .
$$

Lemma 2.4. Let $(x(t), y(t))^{\top}$ be any solution of system (1.5)-(1.6), then

$$
\begin{aligned}
& \liminf _{t \rightarrow+\infty} x(t) \geqslant \frac{a_{1}^{L} k_{1}^{L}}{c_{1}^{M}\left(1-m^{L}\right)^{2} M_{2}+k_{1}^{L} b^{M}} \stackrel{\text { def }}{=} m_{1}, \\
& \liminf _{t \rightarrow+\infty} y(t) \geqslant \frac{r_{2}^{L}\left(\left(1-m^{M}\right) m_{1}+k_{2}^{L}\right)}{c_{2}^{M}} \stackrel{\text { def }}{=} m_{2} .
\end{aligned}
$$

Proof. For $\varepsilon$ small enough, it follows from (2.5) that there exists a $T_{2}>T_{1}$ such that

$$
y(t)<M_{2}+\varepsilon, \quad \forall t \geqslant T_{2}
$$

Let $(x(t), y(t))^{T}$ be any solution of system (1.5)-(1.6). For $t>T_{2},(2.7)$ together with the first equation of system (1.5) leads to

$$
\begin{aligned}
\dot{x}(t) & =x\left(a_{1}(t)-b(t) x\right)-\frac{c_{1}(t)(1-m(t))^{2} x^{2} y}{(1-m(t))^{2} x^{2}+k_{1}(t)} \\
& \geqslant x\left(a_{1}(t)-b(t) x\right)-\frac{c_{1}(t)(1-m(t))^{2} x^{2} y}{k_{1}(t)} \\
& \geqslant x\left(a_{1}^{L}-\frac{c_{1}^{M}\left(1-m^{L}\right)^{2}\left(M_{2}+\varepsilon\right)}{k_{1}^{L}} x-b^{M} x\right) .
\end{aligned}
$$

Applying Lemma 2.1 to (2.8), it immediately follows that

$$
\liminf _{t \rightarrow+\infty} x(t) \geqslant \frac{a_{1}^{L}}{\frac{c_{1}^{M}\left(1-m^{L}\right)^{2}\left(M_{2}+\varepsilon\right)}{k_{1}^{L}}+b^{M}} .
$$

Setting $\varepsilon \rightarrow 0$, then

$$
\liminf _{t \rightarrow+\infty} x(t) \geqslant \frac{a_{1}^{L} k_{1}^{L}}{c_{1}^{M}\left(1-m^{L}\right)^{2} M_{2}+k_{1}^{L} b^{M}} \stackrel{\text { def }}{=} m_{1} .
$$

Let $\varepsilon>0$ be any positive constant small enough such that $\varepsilon<\frac{1}{2} \mathrm{~m}_{1}$. It then follows from (2.9) that there 
exists a $T_{3}>T_{2}$ such that

$$
x(t)>m_{1}-\varepsilon, \quad \forall t \geqslant T_{3} .
$$

From the second equation of system (1.5) and (2.10), we have

$$
\begin{aligned}
\dot{y}(t) & =y\left(a_{2}(t)-\frac{c_{2}(t) y}{(1-m(t)) x+k_{2}(t)}\right) \\
& \geqslant y(t)\left[a_{2}^{L}-\frac{c_{2}^{M} y(t)}{\left(1-m^{M}\right)\left(m_{1}-\varepsilon\right)+k_{2}^{L}}\right] .
\end{aligned}
$$

Applying Lemma 2.1 to (2.11), then

$$
\liminf _{t \rightarrow+\infty} y(t) \geqslant \frac{a_{2}^{L}\left(\left(1-m^{M}\right)\left(m_{1}-\varepsilon\right)+k_{2}^{L}\right)}{c_{2}^{M}} .
$$

Setting $\varepsilon \rightarrow 0$ in above inequality leads to

$$
\liminf _{t \rightarrow+\infty} y(t) \geqslant \frac{a_{2}^{L}\left(\left(1-m^{M}\right) m_{1}+k_{2}^{L}\right)}{c_{2}^{M}} \stackrel{\text { def }}{=} m_{2} .
$$

As a direct corollary of Lemma 2.3 and (2.4), we have:

Theorem 2.5. Under the assumption $\left(\mathrm{H}_{1}\right)$ holds, system (1.5)-(1.6) is permanent.

Remark 2.6. Noting that conditions of Theorem 2.5 is independent of the prey refuge which means that prey refuge has no influence on the persistent property of the system.

\section{Global attractivity}

Before we state the main result of this section, we introduce some notations. Set

$$
\begin{aligned}
\Delta_{1}\left(m_{1}\right) & \stackrel{\text { def }}{=}\left((1-m(t))^{2} m_{1}^{2}+k_{1}(t)\right)^{2}, \\
\Delta_{1}\left(M_{1}\right) & \stackrel{\text { def }}{=}\left((1-m(t))^{2} M_{1}^{2}+k_{1}(t)\right)^{2}, \\
\Delta_{2}\left(m_{1}\right) & \stackrel{\text { def }}{=}\left((1-m(t)) m_{1}+k_{2}(t)\right)^{2}, \\
A_{1}(t) & \stackrel{\text { def }}{=} b(t)+\frac{c_{1}(t)(1-m(t))^{2} k_{1}(t) m_{2}}{\Delta_{1}\left(M_{1}\right)} \\
& -\frac{c_{1}(t)(1-m(t))^{4} M_{2} M_{1}^{2}}{\Delta_{1}\left(m_{1}\right)}-\frac{c_{2}(t)(1-m(t)) M_{2}}{\Delta_{2}\left(m_{1}\right)}, \\
A_{2}(t) & \stackrel{\text { def }}{=} \frac{c_{2}(t) m_{2}}{(1-m(t)) M_{2}+k_{2}(t)}-\frac{c_{1}(t)(1-m(t))^{2} M_{1}}{(1-m(t))^{2} m_{1}^{2}+k_{1}(t)} .
\end{aligned}
$$

Theorem 3.1. In addition to $\left(\mathrm{H}_{1}\right)$, assume further that

$$
\liminf _{t \rightarrow+\infty}\left\{A_{1}(t), A_{2}(t)\right\}>0,
$$

then for any positive solutions $(x(t), y(t))^{\top}$ and $\left(x_{1}(t), y_{1}(t)\right)^{\top}$ of system (1.5), one has

$$
\lim _{t \rightarrow+\infty}\left(\left|x(t)-x_{1}(t)\right|+\left|y(t)-y_{1}(t)\right|\right)=0 .
$$


Noting that conditions of Theorem 3.1 are all depend on time $t$, and this in some cases maybe not be easy to verify, in this case we could use the following corollary to verify the stability property of the system.

Corollary 3.2. In addition to (2.6), assume further that

$$
A_{1}>0, \quad A_{2}>0,
$$

then for any positive solutions $(x(t), y(t))^{\top}$ and $\left(x_{1}(t), y_{1}(t)\right)^{\top}$ of system (1.5), one has

$$
\lim _{t \rightarrow+\infty}\left(\left|x(t)-x_{1}(t)\right|+\left|y(t)-y_{1}(t)\right|\right)=0,
$$

where

$$
\begin{aligned}
& A_{1} \stackrel{\text { def }}{=} b^{L}+\frac{c_{1}^{L}\left(1-m^{M}\right)^{2} k_{1}^{L} m_{2}}{\left(\left(1-m^{L}\right)^{2} M_{1}^{2}+k_{1}^{M}\right)^{2}} \\
&-\frac{c_{1}^{M}\left(1-m^{L}\right)^{4} M_{2} M_{1}^{2}}{\left(\left(1-m^{M}\right)^{2} m_{1}^{2}+k_{1}^{L}\right)^{2}}-\frac{c_{2}^{M}\left(1-m^{L}\right) M_{2}}{\left(\left(1-m^{M}\right) m_{1}+k_{1}^{L}\right)^{2}}, \\
& A_{2} \stackrel{\text { def }}{=} \frac{c_{2}^{L} m_{2}}{\left(1-m^{L}\right) M_{2}+k_{2}^{M}}-\frac{c_{1}^{M}\left(1-m^{L}\right)^{2} M_{1}}{\left(1-m^{M}\right)^{2} m_{1}^{2}+k_{1}^{L}} .
\end{aligned}
$$

One may be interesting in seeking the influence of prey refuge on the stability property of the system as a direct consequence of Corollary 3.2, we have:

Corollary 3.3. In addition to (2.6), assume further that

$$
A_{3}>0, \quad A_{4}>0,
$$

then for any positive solutions $(x(t), y(t))^{\top}$ and $\left(x_{1}(t), y_{1}(t)\right)^{\top}$ of system (1.5), one has

$$
\lim _{t \rightarrow+\infty}\left(\left|x(t)-x_{1}(t)\right|+\left|y(t)-y_{1}(t)\right|\right)=0,
$$

where

$$
\begin{aligned}
& A_{3} \stackrel{\text { def }}{=} b^{\mathrm{L}}-\frac{c_{1}^{M}\left(1-m^{\mathrm{L}}\right)^{4} M_{2} M_{1}^{2}}{\left(k_{1}^{\mathrm{L}}\right)^{2}}-\frac{c_{2}^{M}\left(1-m^{\mathrm{L}}\right) M_{2}}{\left(k_{1}^{\mathrm{L}}\right)^{2}}, \\
& A_{4} \stackrel{\text { def }}{=} \frac{c_{2}^{\mathrm{L}} m_{2}}{M_{2}+k_{2}^{M}}-\frac{c_{1}^{M}\left(1-m^{\mathrm{L}}\right)^{2} M_{1}}{k_{1}^{\mathrm{L}}} .
\end{aligned}
$$

Remark 3.4. Noting that if $m(t)$ is large enough, then $A_{3}>0, A_{4}>0$ always hold and consequently, predator and prey species could be coexistence in a stable state. This means that prey refuge has positive effect to the stability property of the system.

Proof of Theorem 3.1. Condition (3.1) implies that there exists an enough small positive constant $\varepsilon$ (without loss of generality, we may assume that $\left.\varepsilon<\frac{1}{2}\left\{m_{1}, m_{2}\right\}\right)$ such that

$$
\begin{aligned}
A_{1}(\varepsilon, t)= & b(t)+\frac{c_{1}(t)(1-m(t))^{2} k_{1}(t)\left(m_{2}-\varepsilon\right)}{\Delta_{1}\left(M_{1}^{\varepsilon}\right)} \\
& -\frac{c_{1}(t)(1-m(t))^{4}\left(M_{2}+\varepsilon\right)\left(M_{1}+\varepsilon\right)^{2}}{\Delta_{1}\left(m_{1}^{\varepsilon}\right)} \\
& -\frac{c_{2}(t)(1-m(t))\left(M_{2}+\varepsilon\right)}{\Delta_{2}\left(m_{1}^{\varepsilon}\right)} \geqslant \varepsilon,
\end{aligned}
$$




$$
\begin{aligned}
A_{2}(\varepsilon, t)= & \frac{c_{2}(t)\left(m_{2}-\varepsilon\right)}{(1-m(t))\left(M_{2}+\varepsilon\right)+k_{2}(t)} \\
& -\frac{c_{1}(t)(1-m(t))^{2}\left(M_{1}+\varepsilon\right)}{(1-m(t))^{2}\left(m_{1}-\varepsilon\right)^{2}+k_{1}(t)} \geqslant \varepsilon,
\end{aligned}
$$

where

$$
\begin{aligned}
& \Delta_{1}\left(m_{1}^{\varepsilon}\right) \stackrel{\text { def }}{=}\left((1-m(t))^{2}\left(m_{1}-\varepsilon\right)^{2}+k_{1}(t)\right)^{2}, \\
& \Delta_{1}\left(M_{1}^{\varepsilon}\right) \stackrel{\text { def }}{=}\left((1-m(t))^{2}\left(M_{1}+\varepsilon\right)^{2}+k_{1}(t)\right)^{2}, \\
& \Delta_{2}\left(m_{1}^{\varepsilon}\right) \stackrel{\text { def }}{=}\left((1-m(t))\left(m_{1}-\varepsilon\right)+k_{2}(t)\right)^{2} .
\end{aligned}
$$

For two arbitrary positive solutions $(x(t), y(t))^{\top}$ and $\left(x_{1}(t), y_{1}(t)\right)^{\top}$ of system (1.5). For above $\varepsilon>0$, it then follows from (2.2), (2.5), (2.10) and (2.12) that there exists a $T>T_{3}$ such that for all $t \geqslant T$,

$$
\begin{aligned}
& x(t), x_{1}(t)<M_{1}+\varepsilon, \quad y(t), y_{1}(t)<M_{2}+\varepsilon, \\
& x(t), x_{1}(t)>m_{1}-\varepsilon, \quad y(t), y_{1}(t)>m_{2}-\varepsilon .
\end{aligned}
$$

Set

$$
\begin{aligned}
& \Delta_{1}\left(x(t), x_{1}(t)\right)=\left((1-m(t))^{2}\left(x_{1}(t)\right)^{2}+k_{1}(t)\right)\left((1-m(t))^{2}(x(t))^{2}+k_{1}(t)\right), \\
& \Delta_{2}\left(x(t), x_{1}(t)\right)=\left((1-m(t)) x(t)+k_{2}(t)\right)\left((1-m(t)) x_{1}(t)+k_{2}(t)\right) .
\end{aligned}
$$

Now we let

$$
\begin{aligned}
& V_{1}(t)=\left|\ln x(t)-\ln x_{1}(t)\right|, \\
& V_{2}(t)=\left|\ln y(t)-\ln y_{1}(t)\right| .
\end{aligned}
$$

Then for $t>T$, we have

$$
\begin{aligned}
D^{+} V_{1}(t) \leqslant & \operatorname{sgn}\left(x(t)-x_{1}(t)\right)\left(-b(t) x(t)-\frac{c_{1}(t)(1-m(t))^{2} x(t) y(t)}{(1-m(t))^{2}(x(t))^{2}+k_{1}(t)}\right. \\
& \left.\left.+b(t) x_{1}(t)\right)+\frac{c_{1}(t)(1-m(t))^{2} x_{1}(t) y_{1}(t)}{(1-m(t))^{2}\left(x_{1}(t)\right)^{2}+k_{1}(t)}\right) \\
\leqslant & -b(t)\left|x(t)-x_{1}(t)\right|+\operatorname{sgn}\left(x(t)-x_{1}(t)\right) c_{1}(t)(1-m(t))^{2} \\
& \times\left(-\frac{x(t) y(t)}{(1-m(t))^{2}(x(t))^{2}+k_{1}(t)}+\frac{x(t) y_{1}(t)}{(1-m(t))^{2}(x(t))^{2}+k_{1}(t)}\right. \\
& \left.-\frac{x(t) y_{1}(t)}{(1-m(t))^{2}(x(t))^{2}+k_{1}(t)}+\frac{x_{1}(t) y_{1}(t)}{(1-m(t))^{2}\left(x_{1}(t)\right)^{2}+k_{1}(t)}\right) \\
\leqslant & -b(t)\left|x(t)-x_{1}(t)\right|+\frac{c_{1}(t)(1-m(t))^{2} x(t)}{(1-m(t))^{2}(x(t))^{2}+k_{1}(t)}\left|y(t)-y_{1}(t)\right| \\
& +\operatorname{sgn}\left(x(t)-x_{1}(t)\right) c_{1}(t)(1-m(t))^{2} y_{1}(t) \\
& \times\left(-\frac{x(t)}{(1-m(t))^{2}(x(t))^{2}+k_{1}(t)}+\frac{x_{1}(t)}{(1-m(t))^{2}\left(x_{1}(t)\right)^{2}+k_{1}(t)}\right) \\
\leqslant & -b(t)\left|x(t)-x_{1}(t)\right|+\frac{c_{1}(t)(1-m(t))^{2} x(t)}{(1-m(t))^{2}(x(t))^{2}+k_{1}(t)}\left|y(t)-y_{1}(t)\right| \\
& -\frac{c_{1}(t)(1-m(t))^{2} k_{1}(t) y_{1}(t)}{\Delta_{1}\left(x(t), x_{1}(t)\right)}\left|x(t)-x_{1}(t)\right| \\
& +\operatorname{sgn}\left(x(t)-x_{1}(t)\right) \frac{c_{1}(t)(1-m(t))^{4} y_{1}(t) x(t) x_{1}(t)}{\Delta_{1}\left(x(t), x_{1}(t)\right)}\left(-x_{1}(t)+x(t)\right) \\
\leqslant & -b(t)\left|x(t)-x_{1}(t)\right|+\frac{c_{1}(t)(1-m(t))^{2} x(t)}{(1-m(t))^{2}(x(t))^{2}+k_{1}(t)}\left|y(t)-y_{1}(t)\right| \\
&
\end{aligned}
$$




$$
\begin{aligned}
& -\frac{c_{1}(t)(1-m(t))^{2} k_{1}(t) y_{1}(t)}{\Delta_{1}\left(x(t), x_{1}(t)\right)}\left|x(t)-x_{1}(t)\right| \\
& +\frac{c_{1}(t)(1-m(t))^{4} y_{1}(t) x(t) x_{1}(t)}{\Delta_{1}\left(x(t), x_{1}(t)\right)}\left|x(t)-x_{1}(t)\right| \\
& \leqslant-b(t)\left|x(t)-x_{1}(t)\right|+\frac{c_{1}(t)(1-m(t))^{2}\left(M_{1}+\varepsilon\right)}{\left.(1-m(t))^{2}\left(m_{1}-\varepsilon\right)\right)^{2}+k_{1}(t)}\left|y(t)-y_{1}(t)\right| \\
& -\frac{c_{1}(t)(1-m(t))^{2} k_{1}(t)\left(m_{2}-\varepsilon\right)}{\Delta_{1}\left(M_{1}^{\varepsilon}\right)}\left|x(t)-x_{1}(t)\right| \\
& +\frac{c_{1}(t)(1-m(t))^{4}\left(M_{2}+\varepsilon\right)\left(M_{1}+\varepsilon\right)^{2}}{\Delta_{1}\left(m_{1}^{\varepsilon}\right)}\left|x(t)-x_{1}(t)\right|
\end{aligned}
$$

and

$$
\begin{aligned}
D^{+} V_{2}(t)= & \operatorname{sgn}\left(y(t)-y_{1}(t)\right)\left(-\frac{c_{2}(t) y(t)}{(1-m(t)) x(t)+k_{2}(t)}+\frac{c_{2}(t) y_{1}(t)}{(1-m(t)) x_{1}(t)+k_{2}(t)}\right) \\
= & \operatorname{sgn}\left(y(t)-y_{1}(t)\right)\left(-\frac{c_{2}(t) y(t)}{(1-m(t)) x(t)+k_{2}(t)}+\frac{c_{2}(t) y_{1}(t)}{(1-m(t)) x(t)+k_{2}(t)}\right. \\
& \left.-\frac{c_{2}(t) y_{1}(t)}{(1-m(t)) x(t)+k_{2}(t)}+\frac{c_{2}(t) y_{1}(t)}{(1-m(t)) x_{1}(t)+k_{2}(t)}\right) \\
\leqslant & -\frac{c_{2}(t) y(t)}{(1-m(t)) x(t)+k_{2}(t)}\left|y(t)-y_{1}(t)\right| \\
& +\operatorname{sgn}\left(y(t)-y_{1}(t)\right) \frac{c_{2}(t)(1-m(t)) y_{1}(t)}{\Delta_{2}\left(x(t), x_{1}(t)\right)}\left(-x_{1}(t)+x(t)\right) \\
\leqslant & -\frac{c_{2}(t)\left(m_{2}-\varepsilon\right)}{(1-m(t))\left(M_{2}+\varepsilon\right)+k_{2}(t)}\left|y(t)-y_{1}(t)\right| \\
& +\frac{c_{2}(t)(1-m(t))\left(M_{2}+\varepsilon\right)}{\Delta_{2}\left(m_{1}^{\varepsilon}\right)}\left|x(t)-x_{1}(t)\right| .
\end{aligned}
$$

Now let us set

$$
\mathrm{V}(\mathrm{t})=\mathrm{V}_{1}(\mathrm{t})+\mathrm{V}_{2}(\mathrm{t})
$$

Then

$$
\begin{aligned}
D^{+} V(t) \leqslant & -\left(b(t)+\frac{c_{1}(t)(1-m(t))^{2} k_{1}(t)\left(m_{2}-\varepsilon\right)}{\Delta_{1}\left(M_{1}^{\varepsilon}\right)}\right. \\
& -\frac{c_{1}(t)(1-m(t))^{4}\left(M_{2}+\varepsilon\right)\left(M_{1}+\varepsilon\right)^{2}}{\Delta_{1}\left(m_{1}^{\varepsilon}\right)} \\
& \left.-\frac{c_{2}(t)(1-m(t))\left(M_{2}+\varepsilon\right)}{\Delta_{2}\left(m_{1}^{\varepsilon}\right)}\right)\left|x(t)-x_{1}(t)\right| \\
& -\left(\frac{c_{2}(t)\left(m_{2}-\varepsilon\right)}{(1-m(t))\left(M_{2}+\varepsilon\right)+k_{2}(t)}\right. \\
& \left.-\frac{c_{1}(t)(1-m(t))^{2}\left(M_{1}+\varepsilon\right)}{(1-m(t))^{2}\left(m_{1}-\varepsilon\right)^{2}+k_{1}(t)}\right)\left|y(t)-y_{1}(t)\right| \\
= & -A_{1}(\varepsilon, t)\left|x(t)-x_{1}(t)\right|-A_{2}(\varepsilon, t)\left|y(t)-y_{1}(t)\right| .
\end{aligned}
$$

Integrating both sides of (3.3) on interval $[T, t)$, then

$$
V(t)-V(T) \leqslant \int_{T}^{t}\left[-A_{1}(\varepsilon, s)\left|x(s)-x_{1}(s)\right|-A_{2}(\varepsilon, s)\left|y(s)-y_{1}(s)\right|\right] d s, \quad \text { for } t \geqslant T .
$$


It follows from (3.2) that

$$
V(t)+\varepsilon \int_{T}^{t}\left[\left|x(s)-x_{1}(s)\right|+\left|y(s)-y_{1}(s)\right|\right] d s \leqslant V(T), \text { for } t \geqslant T .
$$

Therefore, $\mathrm{V}(\mathrm{t})$ is bounded on $[\mathrm{T},+\infty)$ and also

$$
\int_{T}^{t}\left[\left|x(s)-x_{1}(s)\right|+\left|y(s)-y_{1}(s)\right|\right] d s<+\infty .
$$

By Theorem 2.5, $\left|x(t)-x_{1}(t)\right|,\left|y(t)-y_{1}(t)\right|$ are bounded on $[T,+\infty)$. On the other hand, it is easy to see that $\dot{x}(t), \dot{y}(t), \dot{x}_{1}(t)$ and $\dot{y}_{1}(t)$ are bounded for $t \geqslant T$. Therefore, $\left|x(t)-x_{1}(t)\right|,\left|y(t)-y_{1}(t)\right|$ are uniformly continuous on $[\mathrm{T},+\infty)$. By [3, Barbălat Lemma], one can conclude that

$$
\lim _{t \rightarrow+\infty}\left[\left|x(t)-x_{1}(t)\right|+\left|y(t)-y_{1}(t)\right|\right]=0 .
$$

This ends the proof of Theorem 3.1.

\section{Numeric example}

Now let us consider the following example.

Example 4.1.

$$
\begin{aligned}
& \dot{x}(t)=x\left(39+\sin t-10 x-\frac{2(1-0.5)^{2} x y}{(1-0.5)^{2} x^{2}+9}\right), \\
& \dot{y}(t)=y\left(2+\sin t-\frac{3 y}{(1-0.5) x+1}\right) .
\end{aligned}
$$

Corresponding to system (1.5), one has

$$
\begin{array}{ll}
a_{1}(t)=39+\sin t, \quad b(t)=10, & c_{1}(t)=2, \quad m=0.5, \\
k_{1}(t)=9, & a_{2}(t)=2+\sin t, \quad c_{2}(t)=3, \quad k_{2}(t)=1 .
\end{array}
$$

And so,

$$
M_{1}=\frac{r_{1}^{M}}{b_{1}^{L}}=4, \quad M_{2}=\frac{a_{2}^{M}\left(\left(1-m^{L}\right) M_{1}+k_{2}^{M}\right)}{c_{2}^{L}}=\frac{3(0.5 \times 4+1)}{3}=3 .
$$

By simple computation, one can easily verify that

$$
\begin{aligned}
A_{3} & \stackrel{\text { def }}{=} b^{\mathrm{L}}-\frac{\mathrm{c}_{1}^{\mathrm{M}}\left(1-\mathrm{m}^{\mathrm{L}}\right)^{4} \mathrm{M}_{2} \mathrm{M}_{1}^{2}}{\left(\mathrm{k}_{1}^{\mathrm{L}}\right)^{2}}-\frac{\mathrm{c}_{2}^{\mathrm{M}}\left(1-\mathrm{m}^{\mathrm{L}}\right) \mathrm{M}_{2}}{\left(\mathrm{k}_{1}^{\mathrm{L}}\right)^{2}} \\
& =10-\frac{2 \times \frac{1}{16} \times 3 \times 16}{9^{2}}-\frac{3 \times 0.5 \times 3}{9^{2}}>7>0, \\
A_{4} & \stackrel{\text { def }}{=} \frac{\mathrm{c}_{2}^{\mathrm{L}} \mathrm{m}_{2}}{\mathrm{M}_{2}+\mathrm{k}_{2}^{\mathrm{M}}}-\frac{\mathrm{c}_{1}^{\mathrm{M}}\left(1-\mathrm{m}^{\mathrm{L}}\right)^{2} \mathrm{M}_{1}}{\mathrm{k}_{1}^{\mathrm{L}}} \\
& =\frac{3 \times 0.6}{3+1}-\frac{2 \times 0.25 \times 4}{9} \geqslant 0.4-0.3=0.1>0 .
\end{aligned}
$$

Thus, it follows from Theorem 2.5 and Corollary 3.3 that system (4.1) is permanent and globally attractive, numeric simulations (Figures 1,2) also support this finds. 


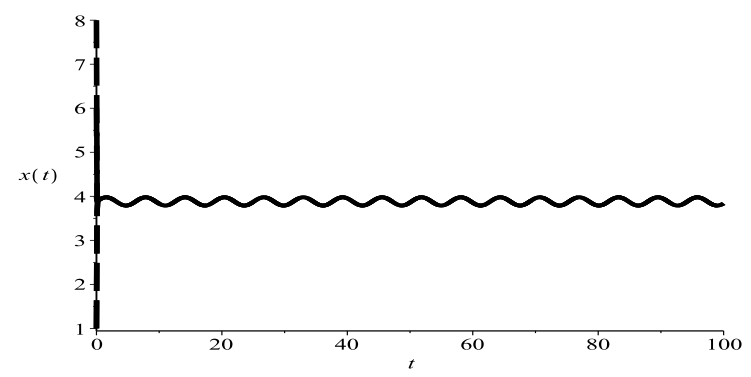

Figure 1: Dynamic behavior of the first component $x(t)$ of the solution $(x(t), y(t))$ of system (4.1) with the initial condition $(x(0), y(0))=(1,2),(1,3),(4,4),(6,5)$ and $(8,1)$, respectively.

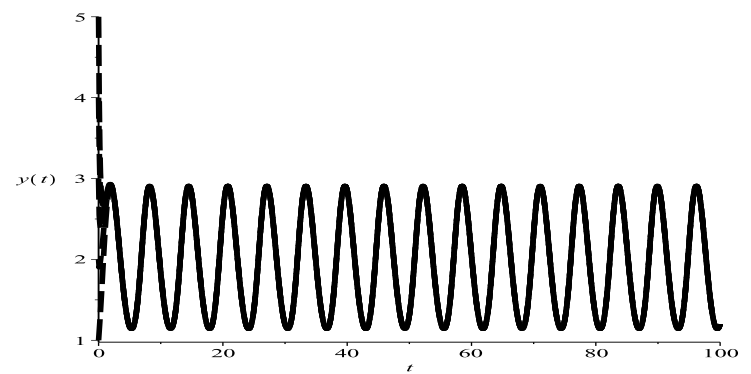

Figure 2: Dynamic behavior of the second component $y(t)$ of the solution $(x(t), y(t))$ of system (4.1) with the initial condition $(x(0), y(0))=(1,2),(1,3),(4,4),(6,5)$ and $(8,1)$, respectively.

\section{Discussion}

In this paper, stimulated by the works of [7] and [36], we propose a predator-prey system incorporating a modified version of the Leslie-Gower functional response as well as that of the Holling-type III functional response and a prey refuge. We pay attention to the nonautonomous case and aim at investigating the persistent and stability property of the system.

One interesting finding is that for system (1.5), the prey refuge has no influence on persistent property of the system, such a finding is similar to that of Chen et al. [7], and different to Yue [36].

We mention here that a more suitable population model should consider the stage structure of the species and it seems interesting to propose and study the stage-structure predator-prey system incorporating a prey refuge, we leave this for future investigation.

\section{Acknowledgment}

The research was supported by the Natural Science Foundation of Fujian Province (2015J01012, 2015J01019).

\section{References}

[1] P. Aguirre, E. González-Olivares, E. Sáez, Two limit cycles in a Leslie-Gower predator-prey model with additive Allee effect, Nonlinear Anal. Real World Appl., 10 (2009), 1401-1416. 1

[2] M. A. Aziz-Alaoui, M. Daher Okiye, Boundedness and global stability for a predator-prey model with modified LeslieGower and Holling-type II schemes, Appl. Math. Lett., 16 (2003), 1069-1075. 1, 1

[3] I. Barbălat, Systémes d'équations diffrentielles d'oscillations non linéaires, (French) Rev. Math. Pures Appl., 4 (1959), 267-270. 3

[4] L.-J. Chen, F.-D. Chen, Global stability of a Leslie-Gower predator-prey model with feedback controls, Appl. Math. Lett., 22 (2009), 1330-1334. 1

[5] L.-J. Chen, F.-D. Chen, Global analysis of a harvested predator-prey model incorporating a constant prey refuge, Int. J. Biomath., 3 (2010), 205-223. 1

[6] L.-J. Chen, F.-D. Chen, L.-J. Chen, Qualitative analysis of a predator-prey model with Holling type II functional response incorporating a constant prey refuge, Nonlinear Anal. Real World Appl., 11 (2010), 246-252. 1

[7] F.-D. Chen, L.-J. Chen, X.-D. Xie, On a Leslie-Gower predator-prey model incorporating a prey refuge, Nonlinear Anal. Real World Appl., 10 (2009), 2905-2908. 1, 5

[8] W.-L. Chen, X.-J. Gong, L. Zhao, H.-Y. Zhang, Dynamics of a nonautonomous discrete Leslie-Gower predator-prey system with a prey refuge, (Chinese) J. Fuzhou Univ. Nat. Sci. Ed., 43 (2015), 6-10. 1

[9] F.-D. Chen, Z. Li, Y.-J. Huang, Note on the permanence of a competitive system with infinite delay and feedback controls, Nonlinear Anal. Real World Appl., 8 (2007), 680-687. 2.1

[10] F.-D. Chen, Y.-M. Wu, Z.-Z. Ma, Stability property for the predator-free equilibrium point of predator-prey systems with a class of functional response and prey refuges, Discrete Dyn. Nat. Soc., 2012 (2012), 5 pages. 1

[11] R. Cressman, J. Garay, A predator-prey refuge system: evolutionary stability in ecological systems, Theor. Popul. Biol., 76 (2009), 248-257. 1

[12] Y.-J. Huang, F.-D. Chen, L. Zhong, Stability analysis of a prey-predator model with Holling type III response function incorporating a prey refuge, Appl. Math. Comput., 182 (2006), 672-683. 1 
[13] L.-L. Ji, C.-Q. Wu, Qualitative analysis of a predator-prey model with constant-rate prey harvesting incorporating a constant prey refuge, Nonlinear Anal. Real World Appl., 11 (2010), 2285-2295. 1

[14] T. K. Kar, S. Misra, Influence of prey reserve in a prey-predator fishery, Nonlinear Anal., 65 (2006), 1725-1735. 1

[15] A. Korobeinikov, A Lyapunov function for Leslie-Gower predator-prey models, Appl. Math. Lett., 14 (2001), 697-699. 1

[16] V. Křivan, On the Gause predator-prey model with a refuge: a fresh look at the history, J. Theoret. Biol., 274 (2011), 67-73. 1

[17] P. H. Leslie, Some further notes on the use of matrices in population mathematics, Biometrika, 35 (1948), 213-245. 1

[18] P. H. Leslie, A stochastic model for studying the properties of certain biological systems by numerical methods, Biometrika, 45 (1958), 16-31. 1

[19] Z. Li, M.-A. Han, F.-D. Chen, Global stability of a stage-structured predator-prey model with modified Leslie-Gower and Holling-type II schemes, Int. J. Biomath., 5 (2012), 13 pages. 1

[20] Z.-H. Ma, S.-F. Wang, W.-D. Li, The effect of prey refuge in a simple predator-prey model, Ecol. Model., 222 (2011), 3453-3454. 1

[21] A. F. Nindjin, M. A. Aziz-Alaoui, M. Cadivel, Analysis of a predator-prey model with modified Leslie-Gower and Hollingtype II schemes with time delay, Nonlinear Anal. Real World Appl., 7 (2007), 1104-1118. 1

[22] Y.-D. Tao, X. Wang, X.-Y. Song, Effect of prey refuge on a harvested predator-prey model with generalized functional response, Commun. Nonlinear Sci. Numer. Simul., 16 (2011), 1052-1059. 1

[23] Y.-M. Wu, F.-D. Chen, W.-L. Chen, Y.-H. Lin, Dynamic behaviors of a nonautonomous discrete predator-prey system incorporating a prey refuge and Holling type II functional response, Discrete Dyn. Nat. Soc., 2012 (2012), 14 pages. 1

[24] Y.-M. Wu, F.-D. Chen, Z.-Z. Ma, Extinction of predator species in a nonautonomous predator-prey system with prey refuge, (Chinese) Appl. Math. J. Chinese Univ. Ser. A, 27 (2012), 359-365.

[25] Y.-M. Wu, F.-D. Chen, Z.-Z. Ma, Y.-H. Lin, Permanence and extinction of a nonautonomous predator-prey system incorporating prey refuge and Rosenzweig functional response, (Chinese) J. Biomath., 29 (2014), 727-731. 1

[26] Y.-M. Wu, W.-L. Chen, H.-Y. Zhang, On permanence and global attractivity of a nonautonomous modified Leslie-Gower predator-prey system incorporating a prey refuge, J. Minjiang Univ., 5 (2012), 13-16. 1

[27] R.-X. Wu, L. Lin, Permanence and global attractivity of the discrete predator-prey system with Hassell-Varley-Holling III type functional response, Discrete Dyn. Nat. Soc., 2013 (2013), 9 pages. 1

[28] R.-X. Wu, L. Zhao, Q.-X. Lin, Stability analysis of a two species amensalism model with Holling II functional response and a cover for the first species, J. Nonlinear Funct. Anal., 2016 (2016), 15 pages. 1

[29] X.-D. Xie, Y.-L. Xue, J.-H. Chen, T.-T. Li, Permanence and global attractivity of a nonautonomous modified Leslie-Gower predator-prey model with Holling-type II schemes and a prey refuge, Adv. Difference Equ., 2016 (2016), 11 pages. 1, 1

[30] R. Yafia, F. El Adnani, H. T. Alaoui, Limit cycle and numerical similations for small and large delays in a predator-prey model with modified Leslie-Gower and Holling-type II schemes, Nonlinear Anal. Real World Appl., 9 (2006), $2055-2067$. 1

[31] W.-S. Yang, Y.-Q. Li, Dynamics of a diffusive predator-prey model with modified Leslie-Gower and Holling-type III schemes, Comput. Math. Appl., 65 (2013), 1727-1737. 1

[32] R.-Z. Yang, C.-R. Zhang, Dynamics in a diffusive predator-prey system with a constant prey refuge and delay, Nonlinear Anal. Real World Appl., 31 (2016), 1-22. 1

[33] R.-Z. Yang, C.-R. Zhang, The effect of prey refuge and time delay on a diffusive predatorprey system with hyperbolic mortality, Complexity, 21 (2016), 446-459. 1

[34] S.-B. Yu, Global asymptotic stability of a predator-prey model with modified Leslie-Gower and Holling-type II schemes, Discrete Dyn. Nat. Soc., 2012 (2012), 8 pages. 1

[35] S.-B. Yu, Global stability of a modified Leslie-Gower model with Beddington-DeAngelis functional response, Adv. Difference Equ., 2014 (2014), 14 pages.

[36] Q. Yue, Dynamics of a modified Leslie-Gower predator-prey model with Holling-type II schemes and a prey refuge, Springerplus, 5 (2016), 12 pages. 1, 5

[37] N. Zhang, F.-D. Chen, Q.-Q. Su, T. Wu, Dynamic behaviors of a harvesting Leslie-Gower predator-prey model, Discrete Dyn. Nat. Soc., 2011 (2011), 14 pages. 1

[38] K.-J. Zhuang, Z.-H. Wen, Dynamical behaviors in a discrete predator-prey model with a prey refuge, Int. J. Comput. Math. Sci., 5 (2011), 195-197. 1 